\title{
Asymmetric truncated Toeplitz operators equal to the zero operator
}

\begin{abstract}
Asymmetric truncated Toeplitz operators are compressions of multiplication operators acting between two model spaces. These operators are natural generalizations of truncated Toeplitz operators. In this paper we describe symbols of asymmetric truncated Toeplitz operators equal to the zero operator.
\end{abstract}

1. Introduction. Let $H^{2}$ denote the Hardy space of the unit disk $\mathbb{D}=$ $\{z:|z|<1\}$, that is, the space of functions analytic in $\mathbb{D}$ with square summable Maclaurin coefficients.

Using the boundary values, one can identify $H^{2}$ with a closed subspace of $L^{2}(\partial \mathbb{D})$, the subspace of functions whose Fourier coefficients with negative indices vanish. The orthogonal projection $P$ from $L^{2}(\partial \mathbb{D})$ onto $H^{2}$, called the Szegö projection, is given by

$$
\operatorname{Pf}(z)=\frac{1}{2 \pi} \int_{0}^{2 \pi} \frac{f\left(e^{i t}\right) d t}{1-e^{-i t} z}, \quad f \in L^{2}(\partial \mathbb{D}) .
$$

Note that if $f \in L^{1}(\partial \mathbb{D})$, then the above integral still defines a function $P f$ analytic in $\mathbb{D}$.

The classical Toeplitz operator $T_{\varphi}$ with symbol $\varphi \in L^{2}(\partial \mathbb{D})$ is defined on $H^{2}$ by

$$
T_{\varphi} f=P(\varphi f) .
$$

2010 Mathematics Subject Classification. 47B32, 47B35, 30H10.

Key words and phrases. Model spaces, truncated Toeplitz operators, asymmetric truncated Toeplitz operators. 
It is known that $T_{\varphi}$ is bounded if and only if $\varphi \in L^{\infty}(\partial \mathbb{D})$. The operator $S=T_{z}$ is called the unilateral shift and its adjoint $S^{*}=T_{\bar{z}}$ is called the backward shift. We have $S f(z)=z f(z)$ and

$$
S^{*} f(z)=\frac{f(z)-f(0)}{z} .
$$

Let $H^{\infty}$ be the algebra of bounded analytic functions on $\mathbb{D}$ and let $\alpha \in$ $H^{\infty}$ be an arbitrary inner function, that is, $|\alpha|=1$ a.e. on $\partial \mathbb{D}$.

By the theorem of A. Beurling (see, for example, [7, Thm. 8.1.1]), every nontrivial, closed $S$-invariant subspace of $H^{2}$ can be expressed as $\alpha H^{2}$ for some inner function $\alpha$. Consequently, every nontrivial, closed $S^{*}$-invariant subspace of $H^{2}$ is of the form

$$
K_{\alpha}=H^{2} \ominus \alpha H^{2}
$$

with $\alpha$ inner. The space $K_{\alpha}$ is called the model space corresponding to $\alpha$.

The kernel function

$$
k_{w}^{\alpha}(z)=\frac{1-\overline{\alpha(w)} \alpha(z)}{1-\bar{w} z}, \quad w, z \in \mathbb{D},
$$

is a reproducing kernel for the model space $K_{\alpha}$, i.e., for each $f \in K_{\alpha}$ and $w \in \mathbb{D}$,

$$
f(w)=\left\langle f, k_{w}^{\alpha}\right\rangle
$$

$\left(\langle\cdot, \cdot\rangle\right.$ being the usual integral inner product). Observe that $k_{w}^{\alpha}$ is a bounded function for every $w \in \mathbb{D}$. It follows that the subspace $K_{\alpha}^{\infty}=K_{\alpha} \cap H^{\infty}$ is dense in $K_{\alpha}$. If $\alpha(w)=0$, then $k_{w}^{\alpha}=k_{w}$, where $k_{w}$ is the Szegö kernel given by $k_{w}(z)=(1-\bar{w} z)^{-1}$.

Let $P_{\alpha}$ denote the orthogonal projection from $L^{2}(\partial \mathbb{D})$ onto $K_{\alpha}$. Then

$$
P_{\alpha} f(z)=\left\langle f, k_{z}^{\alpha}\right\rangle, \quad f \in L^{2}(\partial \mathbb{D}), z \in \mathbb{D} .
$$

Just like with the Szegö projection, $P_{\alpha} f$ is a function analytic in $\mathbb{D}$ for all $f \in L^{1}(\partial \mathbb{D})$.

A truncated Toeplitz operator with a symbol $\varphi \in L^{2}(\partial \mathbb{D})$ is the operator $A_{\varphi}^{\alpha}$ defined on the model space $K_{\alpha}$ by

$$
A_{\varphi}^{\alpha} f=P_{\alpha}(\varphi f) .
$$

Densely defined on bounded functions, the operator $A_{\varphi}^{\alpha}$ can be seen as a compression to $K_{\alpha}$ of the classical Toeplitz operator $T_{\varphi}$ on $H^{2}$.

The study of truncated Toeplitz operators as a class began in 2007 with D. Sarason's paper [13]. In spite of similar definitions, there are many differences between truncated Toeplitz operators and the classical ones. One of the first results from [13] states that, unlike in the classical case, a truncated Toeplitz operator is not uniquely determined by its symbol. More precisely, $A_{\varphi}^{\alpha}=0$ if and only if $\varphi \in \overline{\alpha H^{2}}+\alpha H^{2}$ ([13, Thm. 3.1]). As a consequence, unbounded symbols can produce bounded truncated Toeplitz operators. Moreover, there exist bounded truncated Toeplitz operators for 
which no bounded symbols exist (see [3]). For more interesting results see $[6,9,10,11,12]$.

Recently, the authors in [4] and [5] introduced a generalization of truncated Toeplitz operators, the so-called asymmetric truncated Toeplitz operators. Let $\alpha, \beta$ be two inner functions and let $\varphi \in L^{2}(\partial \mathbb{D})$. An asymmetric truncated Toeplitz operator $A_{\varphi}^{\alpha, \beta}$ is the operator from $K_{\alpha}$ into $K_{\beta}$ given by

$$
A_{\varphi}^{\alpha, \beta} f=P_{\beta}(\varphi f), \quad f \in K_{\alpha} .
$$

The operator $A_{\varphi}^{\alpha, \beta}$ is densely defined. Clearly, $A_{\varphi}^{\alpha, \alpha}=A_{\varphi}^{\alpha}$.

We denote

$$
\mathscr{T}(\alpha, \beta)=\left\{A_{\varphi}^{\alpha, \beta}: \varphi \in L^{2}(\partial \mathbb{D}) \text { and } A_{\varphi}^{\alpha, \beta} \text { is bounded }\right\}
$$

and $\mathscr{T}(\alpha)=\mathscr{T}(\alpha, \alpha)$.

The purpose of this paper is to describe when an operator from $\mathscr{T}(\alpha, \beta)$ is equal to the zero operator. The description is given in terms of the symbol of the operator. This was done in [4] and [5] for the case when $\beta$ divides $\alpha$, that is, when $\alpha / \beta$ is an inner function. It was proved in [4] and [5] that $A_{\varphi}^{\alpha, \beta}=0$ if and only if $\varphi \in \overline{\alpha H^{2}}+\beta H^{2}$. Here we show that this is true for all inner functions $\alpha$ and $\beta$. We also give some examples of rank-one asymmetric truncated Toeplitz operators.

2. Main result. In this section we prove the following.

Theorem 2.1. Let $\alpha, \beta$ be two nonconstant inner functions and let $A_{\varphi}^{\alpha, \beta}$ : $K_{\alpha} \rightarrow K_{\beta}$ be a bounded asymmetric truncated Toeplitz operator with $\varphi \in$ $L^{2}(\partial \mathbb{D})$. Then $A_{\varphi}^{\alpha, \beta}=0$ if and only if $\varphi \in \overline{\alpha H^{2}}+\beta H^{2}$.

We start with a simple technical lemma.

Lemma 2.2. Let $\alpha, \beta$ be two arbitrary inner functions. If

$$
K_{\alpha} \subset \beta H^{2},
$$

then both $\alpha$ and $\beta$ have no zeros in $\mathbb{D}$, or at least one of the functions $\alpha$ or $\beta$ is a constant function.

Proof. Assume that $(2.1)$ holds. If $\beta\left(z_{0}\right)=0$ for some $z_{0} \in \mathbb{D}$, then $f\left(z_{0}\right)=$ 0 for every $f \in K_{\alpha}$. For $f=k_{z_{0}}^{\alpha}$ we get

$$
k_{z_{0}}^{\alpha}\left(z_{0}\right)=\left\|k_{z_{0}}^{\alpha}\right\|^{2}=\frac{1-\left|\alpha\left(z_{0}\right)\right|^{2}}{1-\left|z_{0}\right|^{2}}=0
$$

which implies that $\left|\alpha\left(z_{0}\right)\right|=1$. By the maximum modulus principle, $\alpha$ is a constant function. Hence, the inclusion (2.1) implies that $\beta$ has no zeros in $\mathbb{D}$, or $\alpha$ is a constant function. But (2.1) is equivalent to

$$
K_{\beta} \subset \alpha H^{2} \text {, }
$$

and, by the same reasoning, (2.1) also implies that $\alpha$ has no zeros in $\mathbb{D}$, or $\beta$ is a constant function. This completes the proof. 
Lemma 2.2 can be rephrased as follows. If $\alpha, \beta$ are two nonconstant inner functions and at least one of them has a zero in $\mathbb{D}$, then the inclusion $K_{\alpha} \subset \beta H^{2}$ does not hold. This allows us to prove the following version of Theorem 2.1.

Proposition 2.3. Let $\alpha, \beta$ be two nonconstant inner functions such that each of them has a zero in $\mathbb{D}$ and let $A_{\varphi}^{\alpha, \beta}: K_{\alpha} \rightarrow K_{\beta}$ be a bounded asymmetric truncated Toeplitz operator with $\varphi \in L^{2}(\partial \mathbb{D})$. Then $A_{\varphi}^{\alpha, \beta}=0$ if and only if $\varphi \in \overline{\alpha H^{2}}+\beta H^{2}$.

Proof. The fact that $\varphi \in \overline{\alpha H^{2}}+\beta H^{2}$ implies $A_{\varphi}^{\alpha, \beta}=0$ was proved in $[4$, Thm. 4.3]. For the convenience of the reader we repeat the reasoning from [4].

Assume that $\varphi=\overline{\alpha h}_{1}+\beta h_{2}$ with $h_{1}, h_{2} \in H^{2}$. Then, for every $f \in K_{\alpha}^{\infty}$,

$$
A_{\varphi}^{\alpha, \beta} f=P_{\beta}\left(\overline{\alpha h}_{1} f+\beta h_{2} f\right)=P_{\beta}\left(\overline{\alpha h}_{1} f\right) .
$$

Since $f \perp \alpha H^{2}$, we see that $\overline{\alpha h}_{1} f \perp H^{2}$ and $P_{\beta}\left(\overline{\alpha h}_{1} f\right)=0$. The density of $K_{\alpha}^{\infty}$ implies that $A_{\varphi}^{\alpha, \beta}=0$. Note that this part of the proof does not depend on the existence of zeros of $\alpha$ and $\beta$.

Let us now assume that $A_{\varphi}^{\alpha, \beta}=0$. By the first part of the proof, we can also assume that $\varphi=\bar{\chi}+\psi$ for some $\chi \in K_{\alpha}, \psi \in K_{\beta}$. Let $z_{0} \in \mathbb{D}$ be a zero of $\alpha$. Then $k_{z_{0}}^{\alpha}=k_{z_{0}}$ and

$$
\begin{aligned}
A_{\bar{\chi}}^{\alpha, \beta} k_{z_{0}}^{\alpha} & =P_{\beta}\left(\bar{\chi} k_{z_{0}}\right) \\
& =P_{\beta}\left(\bar{z} \frac{\overline{\chi(z)}-\overline{\chi\left(z_{0}\right)}}{\bar{z}-\overline{z_{0}}}+\overline{\chi\left(z_{0}\right)} k_{z_{0}}\right) \\
& =\overline{\chi\left(z_{0}\right)} k_{z_{0}}^{\beta},
\end{aligned}
$$

because the quotient $\left(\chi(z)-\chi\left(z_{0}\right)\right) /\left(z-z_{0}\right)$ belongs to $K_{\alpha}$ (see [13, Subsection 2.6]).

Hence,

$$
\begin{aligned}
0 & =A_{\varphi}^{\alpha, \beta} k_{z_{0}}^{\alpha}=A_{\bar{\chi}+\psi}^{\alpha, \beta} k_{z_{0}}^{\alpha} \\
& =\overline{\chi\left(z_{0}\right)} k_{z_{0}}^{\beta}+A_{\psi}^{\alpha, \beta} k_{z_{0}}^{\alpha}=P_{\beta}\left[\left(\overline{\chi\left(z_{0}\right)}+\psi\right) k_{z_{0}}\right],
\end{aligned}
$$

which means that

$$
\left(\overline{\chi\left(z_{0}\right)}+\psi\right) k_{z_{0}} \in \beta H^{2}
$$

and, consequently,

$$
\overline{\chi\left(z_{0}\right)}+\psi \in \beta H^{2} .
$$

On the other hand ([4, Lem. 3.2]),

$$
A_{\bar{\psi}+\chi}^{\beta, \alpha}=\left(A_{\bar{\chi}+\psi}^{\alpha, \beta}\right)^{*}=0,
$$


and a similar reasoning can be used to show that if $\beta\left(w_{0}\right)=0, w_{0} \in \mathbb{D}$, then

$$
\chi+\overline{\psi\left(w_{0}\right)} \in \alpha H^{2} .
$$

By (2.2), (2.3) and the first part of the proof we get

$$
A_{\bar{\chi}+\psi\left(w_{0}\right)+\overline{\chi\left(z_{0}\right)}+\psi}^{\alpha, \beta}=0
$$

and

$$
A_{\psi\left(w_{0}\right)+\overline{\chi\left(z_{0}\right)}}^{\alpha, \beta}=-A_{\bar{\chi}+\psi}^{\alpha, \beta}=0 .
$$

From this,

$$
P_{\beta}\left[\left(\psi\left(w_{0}\right)+\overline{\chi\left(z_{0}\right)}\right) f\right]=0
$$

for all $f \in K_{\alpha}$.

If $\psi\left(w_{0}\right)+\overline{\chi\left(z_{0}\right)} \neq 0$, then the above equality means that $P_{\beta}(f)=0$ for all $f \in K_{\alpha}$, that is, $K_{\alpha} \subset \beta H^{2}$. However, by Lemma 2.2, this cannot be the case here. So

$$
\psi\left(w_{0}\right)+\overline{\chi\left(z_{0}\right)}=0
$$

and

$$
\varphi=\bar{\chi}+\psi=\bar{\chi}+\psi\left(w_{0}\right)+\overline{\chi\left(z_{0}\right)}+\psi \in \overline{\alpha H^{2}}+\beta H^{2} .
$$

To give a proof of Theorem 2.1 we use the so-called Crofoot transform. For any inner function $\alpha$ and $w \in \mathbb{D}$, the Crofoot transform $J_{w}^{\alpha}$ is the multiplication operator defined by

$$
J_{w}^{\alpha} f(z)=\frac{\sqrt{1-|w|^{2}}}{1-\bar{w} \alpha(z)} f(z) .
$$

The Crofoot transform $J_{w}^{\alpha}$ is a unitary operator from $K_{\alpha}$ onto $K_{\alpha_{w}}$, where

$$
\alpha_{w}(z)=\frac{w-\alpha(z)}{1-\bar{w} \alpha(z)} .
$$

(see, for example, [8, Thm. 10] and [13, pp. 521-523]). Moreover,

$$
\begin{aligned}
\left(J_{w}^{\alpha}\right)^{*} f & =\left(J_{w}^{\alpha}\right)^{-1} f=J_{w}^{\alpha_{w}} f \\
& =\frac{\sqrt{1-|w|^{2}}}{1-\bar{w} \alpha_{w}} f=\frac{1-\bar{w} \alpha}{\sqrt{1-|w|^{2}}} f .
\end{aligned}
$$

Lemma 2.4. Let $\alpha$ be an inner function and $w \in \mathbb{D}$. For every $z \in \mathbb{D}$ we have

$$
k_{z}^{\alpha_{w}}=\frac{1-|w|^{2}}{(1-w \overline{\alpha(z)})(1-\bar{w} \alpha)} k_{z}^{\alpha} .
$$


Proof. Fix $w, z \in \mathbb{D}$. The reproducing kernel $k_{z}^{\alpha_{w}}$ is given by

$$
k_{z}^{\alpha_{w}}(\lambda)=\frac{1-\overline{\alpha_{w}(z)} \alpha_{w}(\lambda)}{1-\bar{z} \lambda}, \quad \lambda \in \mathbb{D}
$$

Since

$$
\begin{aligned}
1-\overline{\alpha_{w}(z)} \alpha_{w}(\lambda) & =1-\frac{\bar{w}-\overline{\alpha(z)}}{1-w \overline{\alpha(z)}} \frac{w-\alpha(\lambda)}{1-\bar{w} \alpha(\lambda)} \\
& =\frac{\left(1-|w|^{2}\right)(1-\overline{\alpha(z)} \alpha(\lambda))}{(1-w \overline{\alpha(z)})(1-\bar{w} \alpha(\lambda))},
\end{aligned}
$$

we have

$$
\begin{aligned}
k_{z}^{\alpha_{w}}(\lambda) & =\frac{1-|w|^{2}}{(1-w \overline{\alpha(z)})(1-\bar{w} \alpha(\lambda))} \frac{1-\overline{\alpha(z)} \alpha(\lambda)}{1-\bar{z} \lambda} \\
& =\frac{\left(1-|w|^{2}\right)}{(1-w \overline{\alpha(z)})(1-\bar{w} \alpha(\lambda))} k_{z}^{\alpha}(\lambda) .
\end{aligned}
$$

It is known that the map

$$
A \mapsto J_{w}^{\alpha} A\left(J_{w}^{\alpha}\right)^{-1}, \quad A \in \mathscr{T}(\alpha),
$$

carries $\mathscr{T}(\alpha)$ onto $\mathscr{T}\left(\alpha_{w}\right)$ (see [6]). An analogous result can be proved for the asymmetric truncated Toeplitz operators.

Proposition 2.5. Let $\alpha, \beta$ be two inner functions. Let $a, b \in \mathbb{D}$ and let the functions $\alpha_{a}, \beta_{b}$ and the operators $J_{a}^{\alpha}: K_{\alpha} \rightarrow K_{\alpha_{a}}, J_{b}^{\beta}: K_{\beta} \rightarrow K_{\beta_{b}}$ be defined as in (2.5) and (2.4), respectively. If $A$ is a bounded linear operator from $K_{\alpha}$ into $K_{\beta}$, then $A$ belongs to $\mathscr{T}(\alpha, \beta)$ if and only if $J_{b}^{\beta} A\left(J_{a}^{\alpha}\right)^{-1}$ belongs to $\mathscr{T}\left(\alpha_{a}, \beta_{b}\right)$. Moreover, if $A=A_{\varphi}^{\alpha, \beta}$, then $J_{b}^{\beta} A\left(J_{a}^{\alpha}\right)^{-1}=A_{\phi}^{\alpha_{a}, \beta_{b}}$ with

$$
\phi=\frac{(1-\bar{a} \alpha)(1-b \bar{\beta})}{\sqrt{1-|a|^{2}} \sqrt{1-|b|^{2}}} \varphi .
$$

Proof. Let $A$ be a bounded linear operator from $K_{\alpha}$ into $K_{\beta}$. Assume first that $A$ belongs to $\mathscr{T}(\alpha, \beta), A=A_{\varphi}^{\alpha, \beta}$ for $\varphi \in L^{2}(\partial \mathbb{D})$. We show that $J_{b}^{\beta} A\left(J_{a}^{\alpha}\right)^{-1}=A_{\phi}^{\alpha_{a}, \beta_{b}}$ with $\phi$ as in (2.7).

For every $f \in K_{\alpha_{a}}^{\infty}$ and $z \in \mathbb{D}$ we have

$$
\begin{aligned}
J_{b}^{\beta} A_{\varphi}^{\alpha, \beta}\left(J_{a}^{\alpha}\right)^{-1} f(z) & =\frac{\sqrt{1-|b|^{2}}}{1-\bar{b} \beta(z)} P_{\beta}\left(\frac{1-\bar{a} \alpha}{\sqrt{1-|a|^{2}}} \varphi f\right)(z) \\
& =\frac{\sqrt{1-|b|^{2}}}{1-\bar{b} \beta(z)}\left\langle\frac{1-\bar{a} \alpha}{\sqrt{1-|a|^{2}}} \varphi f, k_{z}^{\beta}\right\rangle .
\end{aligned}
$$


By (2.6),

$$
\begin{aligned}
J_{b}^{\beta} A_{\varphi}^{\alpha, \beta}\left(J_{a}^{\alpha}\right)^{-1} f(z) & =\frac{\sqrt{1-|b|^{2}}}{1-\bar{b} \beta(z)}\left\langle\frac{1-\bar{a} \alpha}{\sqrt{1-|a|^{2}}} \varphi f, \frac{(1-\overline{b(z)})(1-\bar{b} \beta)}{1-|b|^{2}} k_{z}^{\beta_{b}}\right\rangle \\
& =\left\langle\frac{1-b \bar{\beta}}{\sqrt{1-|b|^{2}}} \frac{1-\bar{a} \alpha}{\sqrt{1-|a|^{2}}} \varphi f, k_{z}^{\beta_{b}}\right\rangle \\
& =P_{\beta_{b}}\left(\frac{(1-b \bar{\beta})(1-\bar{a} \alpha)}{\sqrt{1-|b|^{2}} \sqrt{1-|a|^{2}}} \varphi f\right)(z) \\
& =A_{\phi}^{\alpha_{a}, \beta_{b}} f(z) .
\end{aligned}
$$

Thus $A \in \mathscr{T}(\alpha, \beta)$ implies that $J_{b}^{\beta} A\left(J_{a}^{\alpha}\right)^{-1} \in \mathscr{T}\left(\alpha_{a}, \beta_{b}\right)$.

To prove the other implication assume that $J_{b}^{\beta} A\left(J_{a}^{\alpha}\right)^{-1}=A_{\phi}^{\alpha_{a}, \beta_{b}} \in$ $\mathscr{T}\left(\alpha_{a}, \beta_{b}\right)$ for some $\phi \in L^{2}(\partial \mathbb{D})$. Then

$$
A=\left(J_{b}^{\beta}\right)^{-1} A_{\phi}^{\alpha_{a}, \beta_{b}} J_{a}^{\alpha}=J_{b}^{\beta_{b}} A_{\phi}^{\alpha_{a}, \beta_{b}}\left(J_{a}^{\alpha_{a}}\right)^{-1} .
$$

But $\left(\alpha_{a}\right)_{a}=\alpha$ and $\left(\beta_{b}\right)_{b}=\beta$, and, by the first part of the proof,

$$
A=J_{b}^{\beta_{b}} A_{\phi}^{\alpha_{a}, \beta_{b}}\left(J_{a}^{\alpha_{a}}\right)^{-1}=A_{\varphi}^{\alpha, \beta}
$$

with

$$
\varphi=\frac{\left(1-\bar{a} \alpha_{a}\right)\left(1-b \overline{\beta_{b}}\right)}{\sqrt{1-|a|^{2}} \sqrt{1-|b|^{2}}} \phi .
$$

Hence, $A \in \mathscr{T}(\alpha, \beta)$. An easy computation shows that $\phi$ satisfies (2.7).

Proof of Theorem 2.1. The fact that $\varphi \in \overline{\alpha H^{2}}+\beta H^{2}$ implies $A_{\varphi}^{\alpha, \beta}=0$ was established in the proof of Proposition 2.3. Assume now that $\varphi \in$ $L^{2}(\partial \mathbb{D})$ and $A_{\varphi}^{\alpha, \beta}=0$.

If $\alpha(0)=\beta(0)=0$, then $\varphi \in \overline{\alpha H^{2}}+\beta H^{2}$ by Proposition 2.3. If $\alpha(0) \neq 0$ or $\beta(0) \neq 0$, put $a=\alpha(0), b=\beta(0)$. By Proposition 2.5,

where

$$
0=J_{b}^{\beta} A_{\varphi}^{\alpha, \beta}\left(J_{a}^{\alpha}\right)^{-1}=A_{\phi}^{\alpha_{a}, \beta_{b}},
$$

$$
\phi=\frac{(1-\bar{a} \alpha)(1-b \bar{\beta})}{\sqrt{1-|a|^{2}} \sqrt{1-|b|^{2}}} \varphi .
$$

Since $\alpha_{a}(0)=\beta_{b}(0)=0$, by Proposition 2.3,

$$
\phi \in \overline{\alpha_{a} H^{2}}+\beta_{b} H^{2} .
$$

Therefore, there exist $h_{1}, h_{2} \in H^{2}$ such that

$$
\frac{(1-\bar{a} \alpha)(1-b \bar{\beta})}{\sqrt{1-|a|^{2}} \sqrt{1-|b|^{2}}} \varphi=\frac{\bar{a}-\bar{\alpha}}{1-a \bar{\alpha}} \bar{h}_{1}+\frac{b-\beta}{1-\bar{b} \beta} h_{2},
$$


and

$$
\varphi=\frac{\bar{a}-\bar{\alpha}}{1-\bar{a} \alpha} \frac{\sqrt{1-|a|^{2}} \sqrt{1-|b|^{2}}}{(1-a \bar{\alpha})(1-b \bar{\beta})} \bar{h}_{1}+\frac{b-\beta}{1-b \bar{\beta}} \frac{\sqrt{1-|a|^{2}} \sqrt{1-|b|^{2}}}{(1-\bar{a} \alpha)(1-\bar{b} \beta)} h_{2} .
$$

Since $|\alpha|=1$ and $|\beta|=1$ on the unit circle $\partial \mathbb{D}$, we have

$$
\frac{\bar{a}-\bar{\alpha}}{1-\bar{a} \alpha}=-\bar{\alpha} \quad \text { and } \quad \frac{b-\beta}{1-b \bar{\beta}}=-\beta .
$$

Consequently,

$$
\varphi=\overline{\alpha g_{1}}+\beta g_{2}
$$

with

$$
g_{1}=-\frac{\sqrt{1-|a|^{2}} \sqrt{1-|b|^{2}}}{(1-\bar{a} \alpha)(1-\bar{b} \beta)} h_{1}, \quad g_{2}=\frac{\sqrt{1-|a|^{2}} \sqrt{1-|b|^{2}}}{(1-\bar{a} \alpha)(1-\bar{b} \beta)} h_{2} .
$$

Since $g_{1}, g_{2} \in H^{2}$, the proof is complete.

Corollary 2.6. If $\varphi$ is in $L^{2}(\partial \mathbb{D})$, then there is a pair of functions $\chi \in K_{\alpha}$, $\psi \in K_{\beta}$, such that $A_{\varphi}^{\alpha, \beta}=A_{\bar{\chi}+\psi}^{\alpha, \beta}$. If $\chi, \psi$ is one such pair, then the most general such pair is of the form $\chi-\bar{c} k_{0}^{\alpha}, \psi+c k_{0}^{\beta}$, with c a scalar.

Proof. The proof is analogous to the proofs given in [13] and [4].

The function $\varphi \in L^{2}(\partial \mathbb{D})$ can be written as $\varphi=\varphi_{+}+\varphi_{-}$with $\varphi_{+}, \bar{\varphi}_{-} \in$ $H^{2}$. If $\chi=P_{\alpha}\left(\bar{\varphi}_{-}\right)$and $\psi=P_{\beta}\left(\varphi_{+}\right)$, then $\varphi-\bar{\chi}-\psi \in \overline{\alpha H^{2}}+\beta H^{2}$. By Theorem 2.1, $A_{\varphi}^{\alpha, \beta}=A_{\bar{\chi}+\psi}^{\alpha, \beta}$

Note that for $f \in K_{\alpha}$,

$$
A_{k_{0}^{\beta}}^{\alpha, \beta} f=P_{\beta}(f-\overline{\beta(0)} \beta f)=P_{\beta} f=A_{1}^{\alpha, \beta} f .
$$

Since $\bar{\alpha} f \perp H^{2}$ for $f \in K_{\alpha}$, we get

$$
A_{\bar{k}_{0}^{\alpha}}^{\alpha, \beta} f=P_{\beta}(f-\alpha(0) \bar{\alpha} f)=P_{\beta} f=A_{1}^{\alpha, \beta} f .
$$

Therefore, if $A_{\varphi}^{\alpha, \beta}=A_{\bar{\chi}+\psi}^{\alpha, \beta}$ with $\chi \in K_{\alpha}, \psi \in K_{\beta}$ as above and $\chi_{1}=\chi-\bar{c} k_{0}^{\alpha}$, $\psi_{1}=\psi+c k_{0}^{\beta}$ for some constant $c \in \mathbb{C}$, then

$$
A_{\bar{\chi}_{1}+\psi_{1}}^{\alpha, \beta}=A_{\bar{\chi}}^{\alpha, \beta}-c A_{1}^{\alpha, \beta}+A_{\psi}^{\alpha, \beta}+c A_{1}^{\alpha, \beta}=A_{\varphi}^{\alpha, \beta} .
$$

Moreover, if $A_{\varphi}^{\alpha, \beta}=A_{\bar{\chi}+\psi}^{\alpha, \beta}=A_{\bar{\chi}_{1}+\psi_{1}}^{\alpha, \beta}$ for any other $\chi_{1} \in K_{\alpha}, \psi_{1} \in K_{\beta}$, then, by Theorem 2.1 , there exist $h_{1}, h_{2} \in H^{2}$ such that

$$
\bar{\chi}+\psi-\bar{\chi}_{1}-\psi_{1}=\overline{\alpha h_{1}}+\beta h_{2} \text {. }
$$

Hence

$$
\psi-\psi_{1}=\beta h_{2}+\overline{\alpha h_{1}+\chi_{1}-\chi}
$$

and

$$
\psi-\psi_{1}=P_{\beta}\left(\psi-\psi_{1}\right)=P_{\beta}\left(\overline{\alpha h_{1}+\chi_{1}-\chi}\right)=c_{1} P_{\beta} 1=c_{1} k_{0}^{\beta}
$$


for some constant $c_{1}$. Similarly,

$$
\chi-\chi_{1}=\alpha h_{1}+\overline{\beta h_{2}+\psi_{1}-\psi}
$$

and

$$
\chi-\chi_{1}=P_{\alpha}\left(\chi-\chi_{1}\right)=P_{\alpha}\left(\overline{\beta h_{2}+\psi_{1}-\psi}\right)=c_{2} k_{0}^{\alpha}
$$

for some constant $c_{2}$.

From this,

$$
\begin{aligned}
0=A_{\bar{\chi}-\bar{\chi}_{1}+\psi-\psi_{1}}^{\alpha, \beta} & =\bar{c}_{2} A_{\bar{k}_{0}^{\alpha}}^{\alpha, \beta}+c_{1} A_{k_{0}^{\beta}}^{\alpha, \beta} \\
& =\left(\bar{c}_{2}+c_{1}\right) A_{1}^{\alpha, \beta}=\left(\bar{c}_{2}+c_{1}\right) P_{\beta \mid K_{\alpha}} .
\end{aligned}
$$

By Lemma 2.2, $\bar{c}_{2}+c_{1}=0$. Putting $c=-c_{1}=\bar{c}_{2}$ we have $\psi_{1}=\psi+c k_{0}^{\beta}$ and $\chi_{1}=\chi-\bar{c} k_{0}^{\alpha}$.

3. Rank-one operators in $\mathscr{T}(\boldsymbol{\alpha}, \boldsymbol{\beta})$. Recall that the model space $K_{\alpha}$ is equipped with a natural conjugation (antilinear, isometric involution) $C_{\alpha}: K_{\alpha} \rightarrow K_{\alpha}$, defined in terms of the boundary values by

$$
C_{\alpha} f(z)=\alpha(z) \bar{z} \overline{f(z)}, \quad|z|=1
$$

(see [13, Subsection 2.3], for more details). A short calculation shows that the conjugate kernel $\widetilde{k}_{w}^{\alpha}=C_{\alpha} k_{w}^{\alpha}$ is given by

$$
\widetilde{k}_{w}^{\alpha}(z)=\frac{\alpha(z)-\alpha(w)}{z-w} .
$$

The function $\alpha$ is said to have a nontangential limit at $\eta \in \partial \mathbb{D}$ if there exists $\alpha(\eta)$ such that $\alpha(z)$ tends to $\alpha(\eta)$ as $z \in \mathbb{D}$ tends to $\eta$ nontangentially (with $|z-\eta| \leq C(1-|z|)$ for some fixed $C>0$ ). We say that $\alpha$ has an angular derivative in the sense of Carathéodory (an ADC) at $\eta \in \partial \mathbb{D}$ if both $\alpha$ and $\alpha^{\prime}$ have nontangential limits at $\eta$ and $|\alpha(\eta)|=1$ (for more details see [9, pp. 33-37]). P. R. Ahern and D. N. Clark proved in $[1,2]$, that $\alpha$ has an ADC at $\eta \in \partial \mathbb{D}$ if and only if every $f \in K_{\alpha}$ has a nontangential limit $f(\eta)$ at $\eta$. If $\alpha$ has an $\mathrm{ADC}$ at $\eta$ and $w$ tends to $\eta$ nontangentially, then the reproducing kernels $k_{w}^{\alpha}$ tend in norm to the function $k_{\eta}^{\alpha} \in K_{\alpha}$ given by (1.1) with $\eta$ in place of $w$. Moreover, $f(\eta)=\left\langle f, k_{\eta}^{\alpha}\right\rangle$ for all $f \in K_{\alpha}$ and

$$
\widetilde{k}_{\eta}^{\alpha}(z)=\frac{\alpha(z)-\alpha(\eta)}{z-\eta}=\alpha(\eta) \bar{\eta} k_{\eta}^{\alpha}(z) .
$$

We can now give some examples of rank-one asymmetric truncated Toeplitz operators (compare with [13, Thm. 5.1]).

Proposition 3.1. Let $\alpha, \beta$ be two nonconstant inner functions.

(a) For $w \in \mathbb{D}$, the operators $\widetilde{k}_{w}^{\beta} \otimes k_{w}^{\alpha}$ and $k_{w}^{\beta} \otimes \widetilde{k}_{w}^{\alpha}$ belong to $\mathscr{T}(\alpha, \beta)$,

$$
\widetilde{k}_{w}^{\beta} \otimes k_{w}^{\alpha}=A_{\frac{\beta(z)}{z-w}}^{\alpha, \beta} \quad \text { and } \quad k_{w}^{\beta} \otimes \widetilde{k}_{w}^{\alpha}=A_{\frac{\alpha(z)}{\bar{z}-\bar{w}}}^{\alpha, \beta} .
$$


(b) If both $\alpha$ and $\beta$ have an $A D C$ at the point $\eta$ of $\partial \mathbb{D}$, then the operator $k_{\eta}^{\beta} \otimes k_{\eta}^{\alpha}$ belongs to $\mathscr{T}(\alpha, \beta)$,

$$
k_{\eta}^{\beta} \otimes k_{\eta}^{\alpha}=A_{k_{\eta}^{\beta}+\bar{k}_{\eta}^{\alpha}-1}^{\alpha, \beta} .
$$

Proof. (a) Let $w \in \mathbb{D}$ and $f \in K_{\alpha}$. Since $\frac{f(z)-f(w)}{z-w} \in K_{\alpha}$ ([13, Subsection 2.6]), we have (for $|z|=1$ )

$$
\begin{aligned}
A_{\frac{\beta(z)}{z-w}}^{\alpha, \beta} f & =P_{\beta}\left(\frac{\beta(z)}{z-w} f(z)\right) \\
& =P_{\beta}\left(\beta(z) \frac{f(z)-f(w)}{z-w}+f(w) \frac{\beta(z)-\beta(w)}{z-w}+f(w) \frac{\beta(w)}{z-w}\right) \\
& =f(w) P_{\beta}\left(\frac{\beta(z)-\beta(w)}{z-w}\right)+f(w) \beta(w) P_{\beta}\left(\frac{\bar{z}}{1-w \bar{z}}\right) \\
& =f(w) \widetilde{k}_{w}^{\beta}=\left\langle f, k_{w}^{\alpha}\right\rangle \widetilde{k}_{w}^{\beta}=\widetilde{k}_{w}^{\beta} \otimes k_{w}^{\alpha}(f) .
\end{aligned}
$$

Similarly,

$$
\begin{aligned}
A_{\frac{\alpha, \beta}{\bar{\alpha}-\bar{w}}}^{\alpha,} f & =P_{\beta}\left(\frac{\overline{\alpha(z)}}{\bar{z}-\bar{w}} f(z)\right)=P_{\beta}\left(\bar{z} \frac{\bar{\alpha}(z) z f(z)}{\bar{z}-\bar{w}}\right)=P_{\beta}\left(\bar{z} \frac{\overline{C_{\alpha} f(z)}}{\bar{z}-\bar{w}}\right) \\
& =P_{\beta}\left(\bar{z} \frac{\overline{C_{\alpha} f(z)}-\overline{C_{\alpha} f(w)}}{\bar{z}-\bar{w}}+\bar{z} \frac{\overline{C_{\alpha} f(w)}}{\bar{z}-\bar{w}}\right)=\overline{C_{\alpha} f(w)} P_{\beta}\left(k_{w}\right) \\
& =\overline{C_{\alpha} f(w)} k_{w}^{\beta}=\overline{\left\langle C_{\alpha} f, k_{w}^{\alpha}\right\rangle} k_{w}^{\beta}=\left\langle f, \widetilde{k}_{w}^{\alpha}\right\rangle k_{w}^{\beta}=k_{w}^{\beta} \otimes \widetilde{k}_{w}^{\alpha}(f) .
\end{aligned}
$$

(b) Let $w \in \mathbb{D}$. Then

$$
A_{k_{w}}^{\alpha, \beta}=A_{k_{w}^{\beta}}^{\alpha, \beta} \quad \text { and } \quad A_{\bar{k}_{w}}^{\alpha, \beta}=A_{\bar{k}_{w}^{\alpha}}^{\alpha, \beta} .
$$

Indeed,

$$
A_{k_{w}^{\beta}}^{\alpha, \beta} f=P_{\beta}\left((1-\overline{\beta(w)} \beta) k_{w} f\right)=P_{\beta}\left(k_{w} f\right)=A_{k_{w}}^{\alpha, \beta} f
$$

for every $f \in K_{\alpha}$. From this, by Lemma 3.2 in [4],

$$
A_{\bar{k}_{w}^{\alpha}}^{\alpha, \beta}=\left(A_{k_{w}^{\alpha}}^{\beta, \alpha}\right)^{*}=\left(A_{k_{w}}^{\beta, \alpha}\right)^{*}=A_{\bar{k}_{w}}^{\alpha, \beta} .
$$

Since for $w \neq 0$ and $|z|=1$,

$$
\begin{aligned}
\frac{\beta(z)}{z-w} & =\frac{\beta(z)-\beta(w)}{z-w}+\frac{\beta(w)}{z-w} \\
& =\widetilde{k}_{w}^{\beta}(z)+\frac{\beta(w)}{w} \cdot \frac{w \bar{z}}{1-w \bar{z}}=\widetilde{k}_{w}^{\beta}(z)+\frac{\beta(w)}{w}\left(\bar{k}_{w}(z)-1\right),
\end{aligned}
$$

we have, by part (a) and (3.1),

$$
\widetilde{k}_{w}^{\beta} \otimes k_{w}^{\alpha}=A_{\frac{\beta(z)}{z-w}}^{\alpha, \beta}=A_{\widetilde{k}_{w}^{\beta}+\frac{\beta(w)}{w}\left(\bar{k}_{w}-1\right)}^{\alpha, \beta}=A_{\widetilde{k}_{w}^{\beta}+\frac{\beta(w)}{w}\left(\bar{k}_{w}^{\alpha}-k_{0}^{\beta}\right)}^{\alpha, \beta} .
$$


If $\alpha$ and $\beta$ have an $A D C$ at $\eta \in \partial \mathbb{D}$, then $k_{w}^{\alpha}$ and $k_{w}^{\beta}$ converge in norm to $k_{\eta}^{\alpha}$ and $k_{\eta}^{\beta}$, respectively, as $w$ tends to $\eta$ nontangentially. Hence $\widetilde{k}_{w}^{\beta} \otimes k_{w}^{\alpha}$ tends to $\widetilde{k}_{\eta}^{\beta} \otimes k_{\eta}^{\alpha}$ in the operator norm. On the other hand,

$$
\widetilde{k}_{w}^{\beta}+\frac{\beta(w)}{w}\left(\bar{k}_{w}^{\alpha}-k_{0}^{\beta}\right) \longrightarrow \widetilde{k}_{\eta}^{\beta}+\frac{\beta(\eta)}{\eta}\left(\bar{k}_{\eta}^{\alpha}-k_{0}^{\beta}\right) \text { in } L^{2}(\partial \mathbb{D}),
$$

which implies that

$$
A_{\widetilde{k}_{w}^{\beta}+\frac{\beta(w)}{w}\left(\bar{k}_{w}^{\alpha}-k_{0}^{\beta}\right)}^{\alpha, \beta} f \longrightarrow A_{\widetilde{k}_{\eta}^{\beta}+\frac{\beta(\eta)}{\eta}\left(\bar{k}_{\eta}^{\alpha}-k_{0}^{\beta}\right)}^{\alpha, \beta} f \text { in } H^{2},
$$

for every $f \in K_{\alpha}^{\infty}$. Therefore,

$$
\widetilde{k}_{\eta}^{\beta} \otimes k_{\eta}^{\alpha}=A_{\widetilde{k}_{\eta}^{\beta}+\frac{\beta(\eta)}{\eta}\left(\bar{k}_{\eta}^{\alpha}-k_{0}^{\beta}\right)} .
$$

Since

we get

$$
\widetilde{k}_{\eta}^{\beta}(z)=\frac{\beta(z)-\beta(\eta)}{z-\eta}=\frac{\beta(\eta)}{\eta} k_{\eta}^{\beta}(z),
$$

$$
\begin{aligned}
k_{\eta}^{\beta} \otimes k_{\eta}^{\alpha} & =\frac{\eta}{\beta(\eta)} \widetilde{k}_{\eta}^{\beta} \otimes k_{\eta}^{\alpha}=\frac{\eta}{\beta(\eta)} A_{\frac{\beta(\eta)}{\eta}\left(k_{\eta}^{\beta}+\bar{k}_{\eta}^{\alpha}-k_{0}^{\beta}\right)}^{\alpha, \beta} \\
& =A_{k_{\eta}^{\beta}+\bar{k}_{\eta}^{\alpha}-k_{0}^{\beta}}^{\alpha, \beta}=A_{k_{\eta}^{\beta}+\bar{k}_{\eta}^{\alpha}-1}^{\alpha, \beta} .
\end{aligned}
$$

It was proved in [13, Thm. 5.1] that the only rank-one operators in $\mathscr{T}(\alpha)$ are the nonzero scalar multiples of the operators $\widetilde{k}_{w}^{\alpha} \otimes k_{w}^{\alpha}, k_{w}^{\alpha} \otimes \widetilde{k}_{w}^{\alpha}$ and $k_{\eta}^{\alpha} \otimes k_{\eta}^{\alpha}$. It is still an open question whether the scalar multiples of the operators from Proposition 3.1 are the only rank-one operators in $\mathscr{T}(\alpha, \beta)$ for arbitrary inner functions $\alpha$ and $\beta$.

\section{REFERENCES}

[1] Ahern, P. R., Clark, D. N., Radial limits and invariant subspaces, Amer. J. Math. 92 (1970), 332-342.

[2] Ahern, P. R., Clark, D. N., Radial n-th derivatives of Blaschke products, Math. Scand. 28 (1971), 189-201.

[3] Baranov, A., Chalendar, I., Fricain, E., Mashreghi, J. E., Timotin, D., Bounded symbols and reproducing kernel thesis for truncated Toeplitz operators, J. Funct. Anal. 259, no. 10 (2010), 2673-2701.

[4] Câmara, C., Jurasik, J., Kliś-Garlicka, K., Ptak, M., Characterizations of asymmetric truncated Toeplitz operators, arXiv:1607.03342.

[5] Câmara, M. C., Partington, J. R., Asymmetric truncated Toeplitz operators and Toeplitz operators with matrix symbol, arXiv:1504.06446.

[6] Cima, J. A., Garcia, S. R., Ross, W. T., Wogen, W. R., Truncated Toeplitz operators: spatial isomorphism, unitary equivalence, and similarity, Indiana Univ. Math. J. 59, no. 2 (2010), 595-620.

[7] Cima, J. A., Matheson, A. L., Ross, W. T., The Cauchy Transform, American Mathematical Society, Providence, RI, 2006. 
[8] Crofoot, R. B., Multipliers between invariant subspaces of the backward shift, Pacific J. Math. 166, no. 2 (1994), 225-246.

[9] Garcia, S. R., Mashreghi, J. E., Ross, W., Introduction to Model Spaces and Their Operators, Cambridge University Press, 2016.

[10] Garcia, S. R., Ross, W. T., A nonlinear extremal problem on the Hardy space, Comput. Methods Funct. Theory 9, no. 2 (2009), 485-524.

[11] Garcia, S. R., Ross, W. T., The norm of truncated Toeplitz operator, CRM Proceedings and Lecture Notes 51 (2010), 59-64.

[12] Garcia, S. R., Ross, W. T., Recent progress on truncated Toeplitz operators, in Blaschke Products and Their Applications, J. Mashreghi, E. Fricain (Eds.), 275-319, Fields Inst. Commun. 65, Springer, New York, 2013.

[13] Sarason, D., Algebraic properties of truncated Toeplitz operators, Operators and Matrices 1, no. 4 (2007), 491-526.

Joanna Jurasik

Department of Mathematics

Maria Curie-Skłodowska University

pl. Marii Curie-Skłodowskiej 1

20-031 Lublin

Poland

e-mail: asia.blicharz@op.pl

Received July 15, 2016
Bartosz Łanucha

Department of Mathematics

Maria Curie-Skłodowska University

pl. Marii Curie-Skłodowskiej 1

20-031 Lublin

Poland

bartosz.lanucha@poczta.umcs.lublin.pl 